\title{
Cost-Effectiveness of the Freeze-All Policy
}

\author{
Matheus Roque ${ }^{1}$, Marcello Valle ${ }^{1}$, Fernando Guimarães ${ }^{1}$, Marcos Sampaio ${ }^{2}$, Selmo Geber ${ }^{2}$ \\ ${ }^{1}$ ORIGEN - Center for Reproductive Medicine, Rio de Janeiro/RJ - Brazil \\ 2ORIGEN - Center for Reproductive Medicine, Belo Horizone/MG - Brazil
}

\begin{abstract}
Objective: To evaluate the cost-effectiveness of freeze-all cycles when compared to fresh embryo transfer.

Methods: This was an observational study with a cost-effectiveness analysis. The analysis consisted of 530 intracytoplasmic sperm injection (ICSI) cycles in a private center in Brazil between January 2012 and December 2013. A total of 530 intracytoplasmic sperm injection (ICSI) cycles - 351 fresh embryo transfers and 179 freeze-all cycles with a gonadotropin-releasing hormone $(\mathrm{GnRH})$ antagonist protocol and day 3 embryo transfers.

Results: The pregnancy rate was $31.1 \%$ in the fresh group and $39.7 \%$ in the freeze-all group. We performed two scenario analyses for costs. In scenario 1 , we included those costs associated with the ICSI cycle (monitoring during controlled ovarian stimulation [COS], oocyte retrieval, embryo transfer, IVF laboratory, and medical costs), embryo cryopreservation of supernumerary embryos, hormone measurements during COS and endometrial priming, medication use (during COS, endometrial priming, and luteal phase support), ultrasound scan for frozen-thawed embryo transfer (FET), obstetric ultrasounds, and miscarriage. The total cost (in USD) per pregnancy was statistically lower in the freeze-all cycles $(19,156.73 \pm$ $1,732.99)$ when compared to the fresh cycles $(23,059.72$ $\pm 2,347.02$ ). Even in Scenario 2, when charging all of the patients in the freeze-all group for cryopreservation (regardless of supernumerary embryos) and for FET, the fresh cycles had a statistically significant increase in treatment costs per ongoing pregnancy.
\end{abstract}

Conclusions: The results presented in this study suggest that the freeze-all policy is a cost-effective strategy when compared to fresh embryo transfer.

Keywords: Cost-effectiveness, Freeze-all, Delayed frozen-thawed embryo transfer

\section{INTRODUCTION}

The first pregnancy described after a frozen-thawed embryo transfer (FET) dates back to 1983 in Australia (Trounson and Mohr, 1983), and the first live birth dates back to 1984 in the Netherlands (Zeilmaker et al., 1984). Since that time, embryo cryopreservation techniques have improved dramatically, and the number of FETs has increased. In 2012, around one-third of all assisted reproductive technology (ART) births reported by the Society for Assisted Reproductive Technology (SART) originated from FET (Doody, 2014).

Today, with improvements in the cryopreservation techniques used, the quality and potential of implantation of frozen embryos are similar to those from fresh embryos (Herrero et al., 2011; Cobo et al., 2012; Wong et al., 2014). With these improvements, and although fresh embryo transfer is still the standard approach in most in vitro fertilization (IVF) centers, there is increasing interest in the freeze-all policy (Pinborg, 2012; Maheshwari and Bhattacharya, 2013; Barnhart, 2014; Shapiro et al., 2014a;).

In the freeze-all policy, the entire cohort of viable embryos is electively cryopreserved and a delayed FET is performed. The potential advantage of this strategy is that the embryo transfer is performed in a more favorable intrauterine environment, possibly improving IVF outcomes (Roque, 2015). Although controlled ovarian stimulation (COS) is an important step during IVF treatment, the supra-physiologic hormone levels observed during COS may lead to morphologic and biochemical endometrial alterations, as well as to endometrial advancement, modifying the endometrial receptivity (ER) and the window of implantation. All of these changes may jeopardize embryo implantation (Horcajadas et al., 2005; Haouzi et al., 2009; Fatemi and Popovic-Todorovic, 2013).

Studies have shown that with the freeze-all policy, better IVF outcomes may be achieved (Shapiro et al., 2011a; Shapiro et al., 2011b; Roque et al., 2013), the risk of early- and/or late-onset ovarian hyperstimulation syndrome (OHSS) is decreased (Devroey et al., 2011), and obstetric and perinatal outcomes are improved (Pinborg et al., 2010; Kaheshwari et al., 2012; Pandey et al., 2012; Pinborg et al., 2013; Wennerholm et al., 2013; Ishihara et al., 2014) when compared to fresh embryo transfers.

However, the freeze-all policy is associated with increased costs during IVF treatments. There are no published studies available in the literature that have evaluated the cost-effectiveness of this strategy. The main goal of this study was to evaluate the cost-effectiveness of freezeall cycles when compared to fresh embryo transfers.

\section{MATERIALS AND METHODS}

In this observational study, we analyzed the database of our previous study (Roque et al., 2015) to evaluate the costs of intracytoplasmic sperm injection (ICSI) cycles with fresh embryo transfer and the freeze-all cycles. The analysis consisted of patients submitted to ART treatment in a private center in Brazil between January 2012 and December 2013. Our Ethics Committee approved the study, and written informed consent was obtained from all patients.

The patients included fulfilled the following inclusion criteria: [1] cleavage-stage day 3 embryo transfers; [2] gonadotropin-releasing hormone $(\mathrm{GnRH})$ antagonist cycles; [3] embryo transfers performed only with good-quality embryos (i.e., $6-10$ cells, with up to $20 \%$ fragmentation and equal blastomere size); and [4] females aged 20-45 years old. The exclusion criteria were: [1] previous recurrent pregnancy loss; [2] implantation failure ( $\geq 3$ previous embryo transfers without pregnancy); [3] antral follicle count (AFC) $\leq 5$; [4] severe male factor infertility (oligospermia <1 million/mL and azoospermia); [5] uterine pathology; and [6] patients with a risk of OHSS - estradiol $>3,000 \mathrm{pg} / \mathrm{mL}$ and/or $>15$ follicles on trigger day.

All the fresh embryo transfers were performed only if progesterone $(P)$ levels were $\leq 1.5 \mathrm{ng} / \mathrm{mL}$ on the trigger day, since progesterone elevation would jeopardize the IVF outcomes in fresh cycles (Papanikolaou et al., 2012; Venetis et al., 2013; Venetis et al., 2015).

The freeze-all strategy was implemented in this study, in cases when $P$ level on the trigger day was > $1.5 \mathrm{ng} / \mathrm{mL}$. 


\section{Controlled ovarian stimulation and oocyte re- trieval}

COS was performed with recombinant follicle-stimulating hormone ( $\mathrm{rFSH}$ ) (Gonal-f®; Merck Serono, Brazil) in a step-down protocol starting on day 2 or 3 of the menstrual cycle. GnRH antagonist (cetrorelix; Cetrotide $\AA$; Merck Serono) was introduced when a leading follicle reached $14 \mathrm{~mm}$. Final oocyte maturation was induced with 250 $\mu \mathrm{g}$ of recombinant human chorionic gonadotropin (hCG) (Ovidrel $®$; Merck Serono) and $0.2 \mathrm{mg}$ of triptorelin (Gonapeptyl daily ${ }^{\circledR}$; Ferring Pharmaceuticals, Inc., Saint-Prex, Switzerland) (Schachter et al., 2008; Decleer et al., 2014) - when at least two follicles reached a diameter of $18 \mathrm{~mm}$. During COS, hormone measurements (luteinizing hormone $[\mathrm{LH}]$, estradiol, and P) were performed on four different days of stimulation, with the last one being performed on the trigger day.

Transvaginal ultrasound-guided oocyte retrieval (all patients were under venous sedation) was performed 35 hours after the trigger with a Wallace 17 Gauge oocyte recovery set (Smiths Medical International Ltd, Kent, UK), followed by ICSI.

Luteal phase support and fresh embryo transfer

The luteal phase support started on the day of oocyte retrieval, with $90 \mathrm{mg}$ of vaginal micronized $\mathrm{P}$ in gel (Crinone $\AA 8 \%$; Merck Serono) for a single daily administration; this was maintained until 9 weeks of pregnancy - if confirmed. Embryo transfers were performed during the cleavage stage (the third day following oocyte retrieval), guided by pelvic ultrasound, using a Labotech soft catheter (Rimmer Brothers, London, UK), and this was only carried out with good-quality embryos.

Freeze-all cycles and freeze-all policy in our reproductive center

In the freeze-all cycles, the good-quality embryos were cryopreserved on day 3 by vitrification, and the FET was also performed on day 3 . Endometrial priming was performed with oral estradiol valerate, which was administered at a dose of $6 \mathrm{mg} /$ day, starting on day 2 of the menstrual cycle. An ultrasound scan and hormone level measurements ( $\mathrm{LH}$, estradiol, and progesterone) were performed after 12 days of estradiol replacement. FET was scheduled when the endometrium was $\geq 7 \mathrm{~mm}$ and the $P$ level was $\leq 1.5$ $\mathrm{ng} / \mathrm{mL}$. If a thinner endometrium was observed, the ultrasound scan and hormone measurements were repeated in 5 days, and if the endometrium remained $<7 \mathrm{~mm}$ and/or the P level was $>1.5 \mathrm{ng} / \mathrm{mL}$, then the embryo transfer was canceled. When the FET was scheduled, the replacement of $\mathrm{P}$ with $90 \mathrm{mg}$ vaginal micronized $\mathrm{P}$ in gel (Crinone $8 \%$; Merck Serono) for a single daily administration started 3 days prior to the embryo transfer. Estradiol valerate and $\mathrm{P}$ were continued up to the 9th week of pregnancy. Embryo transfer was performed under pelvic ultrasound and using the same catheter from the fresh embryo transfers.

\section{Cost analysis}

Cost analysis was performed from the perspective of our private center in Brazil; based on the values that we charge the patients submitted to the freeze-all policy (Scenario 1). The costs included in the analysis were those associated with the ICSI cycle (including monitoring during COS, the oocyte retrieval procedure, embryo transfer, the assisted reproductive laboratory, and medical costs), embryo cryopreservation of the supernumerary embryos, hormone measurements during COS and endometrial priming, medication use (those used during COS, endometrial priming, and lutealphase support), ultrasound scan for FET, obstetric ultrasounds, and miscarriage. All of the prices are expressed in American dollars (USD), and the values were considered when the analysis was performed (February 2015). In our reproductive center, when the freeze-all policy was implemented, we charged the total amount for an ICSI cycle, as described above. However, the cryopreservation costs are charged only for patients with supernumerary embryos; the first FET is free of charge.

We also performed another analysis (Scenario 2) considering that: [1] the two groups (fresh and freeze-all groups) had the same gonadotropin consumption; [2] in the freeze-all group, the ICSI cycle costs did not include embryo transfers, as the patients did not undergo fresh embryo transfer; [3] in the freeze-all group, we charged all patients for the cryopreservation costs, and even patients without a surplus of embryos would pay these costs; and [4] the freeze-all patients were charged for the FET procedure.

\section{Outcomes}

The main outcome measure was the total cost per ongoing pregnancy. Secondary outcomes were: total cost per patient, implantation rates (IR), pregnancy rates (PR), clinical PR (CPR), and ongoing PR (OPR). Ongoing pregnancy was defined as a pregnancy proceeding beyond the 12 th week of gestation. A pregnancy was confirmed with a pregnancy test that was performed 11 days after embryo transfer. A clinical pregnancy was confirmed when a fetal heartbeat was detected by 7-8 weeks gestation.

\section{Statistical analysis}

Data is presented as the mean \pm standard deviation (SD) for continuous variables, or as a percentage for categorical variables. A comparison of the mean values was performed using Student's t-test for independent samples. For a comparison of the categorical data, the chi-squared test or Fisher's exact test was performed when the expected frequency was five or less. The results were considered significant when $P<0.05$. A logistic regression analysis was performed to determine the variables that could be independently associated with ongoing pregnancy, and which could affect the outcomes. Age, basal FSH level, AFC, number of retrieved and mature oocytes, number of 2PN, number of transferred embryos (nET), and cycle type (freeze-all versus fresh) were included in the analysis. The statistical analysis was carried out using the Statistical Package for Social Sciences (SPSS version 19.0 for Windows; Chicago, IL).

\section{RESULTS}

During the study period, we performed 1,357 oocyte retrievals in our IVF center. Of these, 530 patients fulfilled the inclusion criteria and were included in the study. Patients' characteristics and outcomes are depicted on Table 1 . The logistic regression analysis showed that only cycle type (fresh versus freeze-all), age, and the number of transferred embryos, were independently associated with OPR. All other variables were not independently associated with OPR. The odds ratio (OR) for cycle type was 1.73 with a $95 \%$ CI of $1.16-2.58(P=0.008)$, favoring the freeze-all group; the OR for age was 0.90 with a $95 \%$ confidence interval (CI) of $0.858-0.943(P=0.001)$; and the OR for $\mathrm{nET}$ was 1.78 with a $95 \%$ CI of $1.35-2.35(P=0.001)$.

In the treatment cost analysis (values in USD) that we carried out for Scenario 1, we observed that the total cost per ongoing pregnancy was statistically lower in the freeze-all cycles $(19,156.73 \pm 1,732.99)$ when compared to the fresh cycles $(23,059.72 \pm 2,347.02)$. The costs are specified in Table 2. Even in Scenario 2, the fresh cycles had a statistically significant increase in the treatment cost per ongoing pregnancy, as per depicted on Table 3. 
Table 1: Patients' demographic and ICSI outcomes for fresh embryo transfers and freeze-all cycles.

\begin{tabular}{|c|c|c|c|}
\hline & $\begin{array}{l}\text { FRESH } \\
(n=351)\end{array}$ & $\begin{array}{l}\text { FREEZE-ALL } \\
\quad(n=179)\end{array}$ & $\boldsymbol{P}$ \\
\hline Patient age (years) & $35.83 \pm 4.88$ & $35.59 \pm 3.46$ & 0.398 \\
\hline FSH levels (day $2 / 3)(\mathrm{mIU} / \mathrm{mL})$ & $8.44 \pm 2.56$ & $7.96 \pm 2.17$ & 0.023 \\
\hline AFC & $9.23 \pm 3.88$ & $11.46 \pm 4.18$ & 0.001 \\
\hline Days of stimulation & $10.94 \pm 1.18$ & $10.89 \pm 1.09$ & 0.614 \\
\hline Total dose of rFSH (IU) & $2,479.47 \pm 748.47$ & $2,295,77 \pm 696,69$ & 0.006 \\
\hline Days of GnRH antagonist & $5.81 \pm 0.89$ & $5.80 \pm 0.91$ & 0.874 \\
\hline E2 trigger $(\mathrm{pg} / \mathrm{mL})$ & $1,567.88 \pm 649.63$ & $1,636.28 \pm 571.85$ & 0.215 \\
\hline P4 trigger $(\mathrm{ng} / \mathrm{mL})$ & $0.70 \pm 0.27$ & $1.66 \pm 0.14$ & 0.001 \\
\hline Retrieved oocytes & $7.38 \pm 3.94$ & $8.87 \pm 4.14$ & 0.001 \\
\hline MII & $5.39 \pm 3.10$ & $6.28 \pm 3.22$ & 0.002 \\
\hline Fertilization rate & $80.12 \%$ & $76.78 \%$ & 0.042 \\
\hline Post-thaw survival rate & - & $94.9 \%$ & NA \\
\hline $\begin{array}{l}\text { Cycles with supernumerary } \\
\text { embryos }\end{array}$ & $54 \%$ & $68 \%$ & 0.002 \\
\hline nET & $2.27 \pm 0.79$ & $2.07 \pm 0.65$ & 0.003 \\
\hline IR, n (\%) & $159 / 797(19.9 \%)$ & $98 / 370(26.5 \%)$ & 0.011 \\
\hline PR, n (\%) & $144(41 \%)$ & $90(50.3 \%)$ & 0.038 \\
\hline CPR, n (\%) & $126(35.9 \%)$ & $83(46.4 \%)$ & 0.017 \\
\hline OPR, n (\%) & $109(31.1 \%)$ & $71(39.7 \%)$ & 0.044 \\
\hline Miscarriage rate & $19 / 126(15.1 \%)$ & $12 / 83(14.5 \%)$ & 0.900 \\
\hline
\end{tabular}

* Values are expressed as the mean \pm SD or as the absolute number and percentage

FSH - follicle-stimulating hormone; AFC - antral follicle count; rFSH - recombinant follicle-stimulating hormone; GnRH, gonadotropin-releasing hormone; E2 - estradiol; P4 - progesterone; MII - mature eggs; nET - number of transferred embryos; IR - implantation rate; PR - pregnancy rate; CPR - clinical pregnancy rate; OPR - ongoing pregnancy rate

Table 2: Fresh embryo transfer and freeze-all cycle mean costs - cost analysis from the perspective of our private center policy and charges - Scenario 1

\begin{tabular}{|c|c|c|c|}
\hline & $\begin{array}{c}\text { FRESH } \\
(n=351)\end{array}$ & $\begin{array}{l}\text { FREEZE-ALL } \\
\quad(n=179)\end{array}$ & $\boldsymbol{P}$ \\
\hline ICSI cycle & $3,618.50$ & $3,618.50$ & - \\
\hline rFSH & $2,293.65 \pm 692.38$ & $2,123.72 \pm 644.47$ & 0.006 \\
\hline GnRH antagonist & $480.24 \pm 73.58$ & $479.15 \pm 75.53$ & 0.874 \\
\hline Trigger & 111.63 & 111.63 & NA \\
\hline LPS & $268.98 \pm 192.31$ & - & NA \\
\hline EP + LPS & - & $377.30 \pm 211.29$ & NA \\
\hline Hormone measurements - cos & 111.28 & 111.28 & NA \\
\hline Hormone measurements - FET & - & $30.61 \pm 8.39$ & NA \\
\hline US scan - FET & - & $72.72 \pm 19.93$ & NA \\
\hline US - obstetrics & $84.57 \pm 106.26$ & $104.29 \pm 109.02$ & 0.046 \\
\hline Embryo cryopreservation & $416.32 \pm 381.59$ & $521.45 \pm 357.43$ & 0.002 \\
\hline Miscarriage cost & $38.59 \pm 161.54$ & $47.49 \pm 178.79$ & 0.550 \\
\hline Total treatment cost per patient & $7,423.79 \pm 755.59$ & $7,598.48 \pm 686.47$ & 0.010 \\
\hline Treatment cost per ongoing pregnancy & $23,059.72 \pm 2,347.02$ & $19,156.73 \pm 1,732.99$ & 0.001 \\
\hline
\end{tabular}

* Values are expressed as the mean \pm SD, or only the mean when all patients in the group had the same costs; the costs are in USD.

rFSH - recombinant follicle-stimulating hormone; GnRH - gonadotropin-releasing hormone; LPS - luteal phase support; EP - endometrial priming; COS - controlled ovarian stimulation; FET - frozen-thawed embryo transfer; US - ultrasound 


\begin{tabular}{|c|c|c|c|}
\hline & $\begin{array}{c}\text { FRESH } \\
(n=351)\end{array}$ & $\begin{array}{l}\text { FREEZE-ALL } \\
\quad(n=179)\end{array}$ & $\boldsymbol{P}$ \\
\hline ICSI cycle & $3,618.50$ & - & NA \\
\hline $\begin{array}{l}\text { ICSI cycle without embryo } \\
\text { transfer }\end{array}$ & - & $3,270.74$ & NA \\
\hline rFSH & $2,293.65 \pm 692.38$ & $2,293.9$ & NA \\
\hline GnRH antagonist & $480.24 \pm 73.58$ & $479.15 \pm 75.53$ & 0.874 \\
\hline Trigger & 111.63 & 111.63 & NA \\
\hline LPS & $268.98 \pm 192.31$ & - & NA \\
\hline EP + LPS & - & $377.30 \pm 211.29$ & NA \\
\hline Hormone measurements - cos & 111.28 & 111.28 & NA \\
\hline Hormone measurements - FET & - & $30.61 \pm 8.39$ & NA \\
\hline US scan - FET & - & $72.72 \pm 19.93$ & NA \\
\hline US - obstetrics & $84.57 \pm 106.26$ & $104.29 \pm 109.02$ & 0.027 \\
\hline Embryo cryopreservation & $416.32 \pm 381.59$ & 765.08 & 0.001 \\
\hline FET & - & $1,356.29$ & NA \\
\hline Miscarriage cost & $38.59 \pm 161.54$ & $47.49 \pm 178.79$ & 0.374 \\
\hline $\begin{array}{l}\text { Total treatment cost } \\
\text { per patient }\end{array}$ & $7,417.54 \pm 747.95$ & $9,020.58 \pm 399.29$ & 0.001 \\
\hline $\begin{array}{l}\text { Treatment cost per ongoing } \\
\text { pregnancy }\end{array}$ & $23,040.33 \pm 2,323.27$ & $22,742.04 \pm 1,006.66$ & 0.040 \\
\hline
\end{tabular}

\section{DISCUSSION}

To our knowledge, this is the first published study evaluating the freeze-all policy cost-effectiveness. There are various potential benefits of this strategy; however, there is a lack of information regarding its costs. The results presented in this study suggest that the freeze-all policy is a cost-effective strategy when compared to fresh embryo transfer.

With the advancements in reproductive medicine, it is important to individualize IVF treatments and offer the best treatment for each patient to maximize results and decrease treatment risks and costs (La Marca and Sunkara, 2014). It is of fundamental importance that the best approach be performed for each patient, maximizing the results for each embryo transfer, since a great number of patients drop out of the IVF treatments after a single cycle (Verberg et al., 2008). Previous studies have shown that delayed FET is associated with an increase in OPR, and that there are no differences in miscarriage rates (Shapiro et al., 2011a; Shapiro et al., 2011b; Roque et al., 2013; Shapiro et al., 2014; Yang et al., 2015). Our results presented in this study are in agreement with the findings of these aforementioned studies. We observed that even among patients who were submitted to fresh embryo transfers performed only if $P \leq 1.5 \mathrm{ng} / \mathrm{mL}$ on the trigger day, there were advantages favoring the freeze-all policy, with an increase of $33 \%$ and $28 \%$ in implantation and ongoing pregnancy rates, respectively. We did not find statistically significant differences in terms of miscarriage rates between the two groups. It is important to emphasize that our results are based on cleavage-stage embryo transfer; it did not include patients that had all embryos frozen due the risk of OHSS development, as these patients would bring bias to the results; and that all fresh embryo transfers were performed only in patients with $P \leq 1.5 \mathrm{ng} / \mathrm{mL}$ on the trigger day, since progesterone elevation would jeopardize the IVF outcomes in fresh cycles (26-28). (Papanikolaou et al., 2012; Venetis et al., 2013; Santos-Ribeiro et al., 2014; Venetis et al., 2015).

Nowadays, we do not have efficient tools to evaluate in which patients the freeze-all strategy, rather than fresh embryo transfers, should be employed; the only exception appears to be in cases of fertility preservation and among patients with a risk of developing OHSS (Doody, 2014). Thus, it is important to evaluate the cost-effectiveness of the strategy until studies really prove the method's superiority, and until they identify in which patients this procedure should be used. In our study, we found that fresh embryo transfer is around $20 \%$ more expensive for each ongoing pregnancy when compared to the freeze-all strategy. This cost analysis was performed considering the policy employed at our private center in Brazil, as per discussed earlier. While trying to hypothesize a different way to charge the freeze-all patients, including all the possible costs related to this strategy, we performed another analysis (Scenario 2); even in this scenario, the freeze-all policy proved to be a cost-effective strategy. Since our study excluded patients with the risk of developing OHSS, the possible expenses faced by patients that would develop OHSS were not included in our cost analysis (this would increase the mean costs per ongoing pregnancy in the fresh group), and we did not include indirect costs, such as those associated with the extra time spent with patients for FET.

Since this study was not a randomized controlled trial (RCT), it may have some weaknesses. There were differences between the two groups in terms of ovarian re- 
sponse. However, some previous studies (Griesinger et al., 2013; Ji et al., 2013) have shown that in normo-responders, the differences in the number of retrieved oocytes would not interfere with pregnancy outcomes. The patients included in our study fell within a range that was observed in these previous studies. Furthermore, the logistic regression analysis performed in our study showed that the number of oocytes retrieved, the number of mature eggs, and the number of fertilized eggs, were not independently associated with the outcomes. Some questions may be raised, especially regarding the payment policy for the patients who underwent the freeze-all strategy. However, even when we included all the possible costs involved with the freeze-all cycle and the first FET (Scenario 2), this strategy proved to be cost-effective. It seems that the observed increase in OPR in the freeze-all group make up for the possible increase in treatment costs related to this strategy.

This study evaluated issues about the freeze-all cycles that had not been reported in the literature, and reinforce the need for further randomized controlled trials (RCT) pertaining to the freeze-all policy to confirm the effectiveness of this strategy and evaluate its cost-effectiveness. Until now, only four RCTs (Aflatoonian et al., 2010; Shapiro et al., 2011a; Shapiro et al., 2011b; Yang et al., 2015) were published concerning this strategy, and one of them (Aflatoonian et al., 2010) was retracted due to methodological flaws. In conclusion, the results of this study showed that IVF outcomes could be improved when performing the freeze-all policy. It was also determined that this is a cost-effective strategy that can be implemented in our routine practice.

\section{CONFLICT OF INTERESTS}

No conflict of interest have been declared.

\section{Corresponding author:}

Matheus Roque

ORIGEN - Center for Reproductive Medicine

Rio de Janeiro/RJ - Brazil

E-mail: matheusroque@hotmail.com

\section{REFERENCES}

Aflatoonian A, Oskouian H, Ahmedi S, Oskouian L. Can fresh embryo transfers be replaced by cryopreserved-thawed embryo transfers in assisted reproductive cycles? A randomized controlled trial. J Assist Reprod Genet. 2010; 27: 357-63.

Barnhart KT. Introduction: are we ready to eliminate the transfer of fresh embryos in in vitro fertilization? Fertil Steril. 2014; 102: 1-2.

Cobo A, de los Santos MJ, Castellò D, Gámiz P, Campos P, Remohí J. Outcomes of vitrified early cleavage-stage and blastocyst-stage embryos in a cryopreservation program: evaluation of 3,150 warming cycles. Fertil Steril. 2012; 98: 1138-46.

Decleer W, Osmanagaoglu K, Seynhave B, Kolibianakis S, Tarlatzis B, Devroey P. Comparison of hCG triggering versus hCG in combination with GnRH agonist: a prospective randomized controlled trial. Facts Views Vis Obgyn. 2014; 6: 203-9.

Devroey P, Polyzos NP, Blockeel C. An OHSS-Free Clinic by segmentation of IVF treatment. Hum Reprod. 2011; 26: 2593-7.

Doody KJ. Cryopreservation and delayed embryo trans- fer-assisted reproductive technology registry and reporting implications. Fertil Steril. 2014; 102: 27-31.

Fatemi HM, Popovic-Todorovic B. Implantation in assisted reproduction: a look at endometrial receptivity. Reprod Biome Online. 2013; 27: 530-8.

Griesinger G, Mannaerts B, Andersen CY, Witjes H, Kolibianakis EM, Gordon K. Progesterone elevation does not compromise pregnancy rates in high responders: a pooled analysis of in vitro fertilization patients treated with recombinant follicle-stimulating hormone/gonadotropin-releasing hormone antagonist in six trials. Fertil Steril. 2013; 100: $1622-8$.

Haouzi D, Assou S, Mahmoud K, Tondeur S, Reme T, Hedon $B$ et al. Gene expression profile of human endometrial receptivity: comparison between natural and stimulated cycles for the same patients. Hum Reprod. 2009; 24: 143645.

Herrero L, Martínez M, Garcia-Velasco JA. Current status of human oocyte and embryo cryopreservation. Curr Opin Obstet Gynecol. 2011; 23: 245-50.

Horcajadas JA, Riesewijk A, Polman J, van Os R, Pellicer A, Mosselman S, Simón C. Effect of controlled ovarian hyperstimulation in IVF on endometrial gene expression profiles. Mol Hum Reprod. 2005; 11: 195-205.

Ishihara O, Araki R, Kuwuhara A, Itakura A, Saito H, Adamson GD. Impact of frozen-thawed single-blastocyst transfer on maternal and neonatal outcome: an analysis of 277,042 single-embryo transfer cycles from 2008 to 2010 in Japan. Fertil Steril. 2014; 101: 128-33.

Ji J, Liu Y, Tong XH, Luo L, Ma J, Chen Z. The optimum number of oocytes in IVF treatment: an analysis of 2455 cycles in China. Hum Reprod. 2013; 28: 2728-34.

La Marca A, Sunkara SK. Individualization of controlled ovarian stimulation in IVF using ovarian reserve markers: from theory to practice. Hum Reprod Update. 2014; 20 : 124-40.

Maheshwari A, Bhattacharya. Elective frozen replacement cycles for all: ready for prime time? Hum Reprod. 2013; 28: 6-9.

Maheshwari A, Pandey S, Shetty A, Hamilton M, Bhattacharya S. Obstetric and perinatal outcomes in singleton pregnancies resulting from the transfer of frozen thawed versus fresh embryos generated through in vitro fertilization treatment: a systematic review and meta-analysis. Fertil Steril. 2012; 98:368-77.

Pandey S, Shetty A, Hamilton M, Bhattacharya S, Maheshwari A. Obstetric and perinatal outcomes in singleton pregnancies resulting from IVF/ICSI: a systematic review and meta-analysis. Hum Reprod Update. 2012; 18: 485-503.

Papanikolaou EG, Pados G, Grimbizis G, Bili E, Kyriazi L, Polyzos NP, Humaidan P, Tournaye H, Tarlatzis B. GnRH-agonist versus $\mathrm{GnRH}$-antagonist IVF cycles: is the reproductive outcome affected by the incidence of progesterone elevation on the day of HCG triggering? A randomized prospective study. Hum Reprod. 2012; 6: 1822-8.

Pinborg A, Loft A, Aaris Henningsen AK, Rasmussen S, Anderson AN. Infant outcome of 957 singletons born af- 
ter frozen embryo replacement: the Danish National Cohort Study 1995-2006. Fertil Steril. 2010; 94: 1320-7.

Pinborg A. To transfer fresh or thawed embryos? Semin Reprod Med. 2012; 30: 230-5.

Pinborg A, Wennerholm UB, Romundstad LB, Loft A, Aittomaki K, Söderström-Anttila V, Nygren KG, Hazekamp J, Bergh $\mathrm{C}$. Why do singletons conceived after assisted reproduction technology have adverse perinatal outcome? Systematic review and meta-analysis. Hum Reprod Update. 2013; 19 : 87-104.

Roque M, Lattes K, Serra S, Solà I, Geber S, Carreras $R$, Checa MA. Fresh embryo transfer versus frozen embryo transfer in in vitro fertilization cycles: a systematic review and meta-analysis. Fertil Steril. 2013; 99: 156-62.

Roque M. Freeze-all policy: is it time for that? J Assist Reprod Genet 2015a; 32: 171-6.

Roque $M$, Valle $M$, Guimarães $F$, Sampaio $M$, Selmo G. Freeze-all policy: fresh versus frozen-thawed embryo transfer. Fertil Steril. 2015. In press.

Santos-Ribeiro S, Polyzos NP, Haentjens P, Smitz J, Camus $M$, Tournaye $\mathrm{H}$, Blockeel $\mathrm{C}$. Live birth rates after IVF are reduced by both low and high progesterone levels on the day of human chorionic gonadotrophin administration. Hum Reprod 2014; 29: 1698-1705.

Schachter M, Friedler S, Ron-El R, Zimmerman AL, Strassburger D, Bern O, Raziel A. Can pregnancy rate be improved in gonadotropin-releasing hormone (GnRH) antagonist cycles by administering $\mathrm{GnRH}$ agonist before oocyte retrieval? A prospective, randomized study. Fertil Steril. 2008; 90: 1087-93.

Shapiro BS, Daneshmand ST, Garner FC, Aguirre M, Hudson C, Thomas S. Evidence of impaired endometrial receptivity after ovarian stimulation for in vitro fertilization: a prospective randomized trial comparing fresh and frozen-thawed embryo transfer in normal responders. Fertil Steril. 2011a; 96: 344-8.

Shapiro BS, Daneshmand ST, Garner FC, Aguirre M, Hudson C, Thomas S. Evidence of impaired endometrial receptivity after ovarian stimulation for in vitro fertilization: a prospective randomized trial comparing fresh and frozen-thawed embryo transfer in high responders. Fertil Steril. 2011b; 96: 516-8.
Shapiro BS, Daneshmand ST, Garner FC, Aguirre M, Hudson $\mathrm{C}$, Thomas S. Similar ongoing pregnancy rates after blastocyst transfer in fresh donor cycles and autologous cycles using cryopreserved bipronuclear oocytes suggesting similar viability of transferred blastocysts. Fertil Steril. 2010; 93: 319-21.

Shapiro BS, Daneshmand ST, Garner FC, Aguirre M, Hudson C. Clinical rationale for cryopreservation of entire cohorts in lieu of fresh transfer. Fertil Steril. 2014a; 102: 3-9.

Shapiro BS, Daneshmand ST, Garner FC, Aguirre M, Hudson C. Freeze-all can be a superior therapy to another fresh cycle in patients with prior fresh blastocyst implantation failure. Reprod Biomed Online 2014b; 29: 286-90.

Trounson A, Mohr L. Human pregnancy following cryopreservation, thawing and transfer of an eight-cell embryo. Nature. 1983; 305: 707-9.

Venetis CA, Kolibianakis EM, Bosdou JK, Tarlatzis BC. Progesterone elevation and probability of pregnancy after IVF: a systematic review and meta-analysis of over $60000 \mathrm{cy}-$ cles. Hum Reprod Update. 2013; 19: 433-57.

Venetis CA, Kolibianakis EM, Bosdou JK, Lainas GT, Sfontouris IA, Tarlatzis BC, Lainas TG. Estimating the net effect of progesterone elevation on the day of hCG on live birth rates after IVF: a cohort analysis of 3296 IVF cycles. Hum Reprod 2015; 30: 684-91.

Verberg MF, Eijkemans MJ, Heijnen EM, Broekmans FJ, de Klerk C, Fauser BC, Macklon NS. Why do couples drop-out from IVF treatment? A prospective cohort study. Hum Reprod. 2008; 23: 2050-5.

Wennerholm UB, Henningsen AK, Romundstad LB, Bergh C, Pinborg A, Skjaerven R, et al. Perinatal outcomes of chilfren born after frozen-thawed embryo transfer: a Nordic cohort study from the CoNARTaS group. Hum Reprod. 2013; 28: 2545-53.

Yang S, Pang T, Li R, Yang R, Zhen X, Chen X, Wang H, Ma C, Liu P, Qiao J. The individualized choice of embryo transfer timing for patients with elevated serum progesterone level on the HCG day in IVF/ICSI cycles: a prospective randomized clinical study. Gynecol Endocrinol. 2015. In press.

Zeilmaker GH, Alberda AT, van Gent I, Rijkmans CM, Drogendijk AC. Two pregnancies following transfer of intact frozen-thawed embryos. Fertil Steril. 1984; 42: 293-6. 